\section{A six-channel PDP-8 interface}

\section{for nerve impulse data*}

\section{EARL J. KLETSKY}

Laboratory of Sensory Communication

Syracuse University, Syracuse, New York 13210

An interface operating on the $1 / O$ bus of the LINC- 8 has been built which accepts up to six channels of nerve impulse data simultaneously at rates up to $4,000 \mathrm{pps} / \mathrm{channel}$. By means of a $100-\mathrm{KHz}$ clock and a 17-bit counter, the time of occurrence of each nerve impulse is strobed into one of six 17-bit buffer registers. The strobe causes a program interrupt to occur. Two $I / O$ instructions are used to read the buffers into the PDP-8 accumulator. Additional instructions for skipping on data or overflow, enabling the counter, clearing the overflow flag, and clearing the counter are available.

\section{INTRODUCTION}

The LINC-8 computer at the L a boratory of Sensory Communication is used primarily for on-line collection and processing of nerve impulse data from both biological preparations and from real-time electronic analog models of sensory systems. Basic data from all these experiments are temporal trains of voltage pulses derived from actual or simulated nerve impulses by means of level discrimination and pulse shaping. Extracted from this data is the time of occurrence of each pulse with respect to a reference. The resulting times are stored by the computer, processed in a variety of ways, and the results presented to the $\mathrm{E}$ by means of Teletype printout, oscilloscope display, and digital plotter.

It will be noted that only the pertinent data (times of occurrence) are stored in digital form. This has two major advantages over sampling and storing the entire biological waveform. First, there is at least an order of magnitude saving in the amount of digital storage required. Secondly, access to the stored raw data for off-line reprocessing is accomplished very rapidiy. This is in contrast to scanning in real time the biological waveform as it appears stored on analog tape.

The interface described below couples the experiment to the computer. It has been designed and built to accept up to six channels of discriminated nerve impulse data at rates up to $4,000 \mathrm{pps} / \mathrm{channel}$. Temporal resolution between any two channels can be as small as 10 microsec.

*The interface was constructed by the Special Systems Group of the Digital Equipment Corporation following a preliminary design prepared by the author. This work was supported by Grant NINDB-NB-03950-07 from the National Institutes of Health.

\section{THE INTERFACE}

A block diagram of the interface is shown in Fig. 1. It operates as a peripheral device on the $\mathrm{I} / \mathrm{O}$ bus of the computer in conjunction with the program interrupt facility. One of four selectable clock sources drives a 17 -bit counter. When an input pulse occurs on a given channel, the contents of the counter are strobed into a buffer register assigned to a channel, the buffer flag is set, and a program interrupt occurs. When the software skip-chain determines that the interface is responsible for the interrupt, two $I / O$ instructions are used to read the contents of the buffer into the accumulator. If more than one buffer has been loaded, a hard-wired priority chain selects which of the six buffers is to be read. The first $1 / O$ instruction reads the 12 least significant bits into the accumulator. The second instruction is used to read the most significant 5 bits of the buffer as well as a 3-bit hard-wired channel identification code. This instruction also clears the buffer and its flag.

An interrupt is made to occur whenever the 17-bit counter overflows. The associated overflow flag can be cleared by an I/O instruction.

The interface can be enabled either under program control or externally. An 1/O instruction is used to set or clear an enable flip-flop, depending on the contents of Accumulator Bit 11. The enable flip-flop can also be controlled by means of an externally applied voltage level.

A novel $1 / O$ instruction is available which generates a maintenance pulse. The pulse, imitating an input, is applied to all six input channels simultaneously. This capability is used

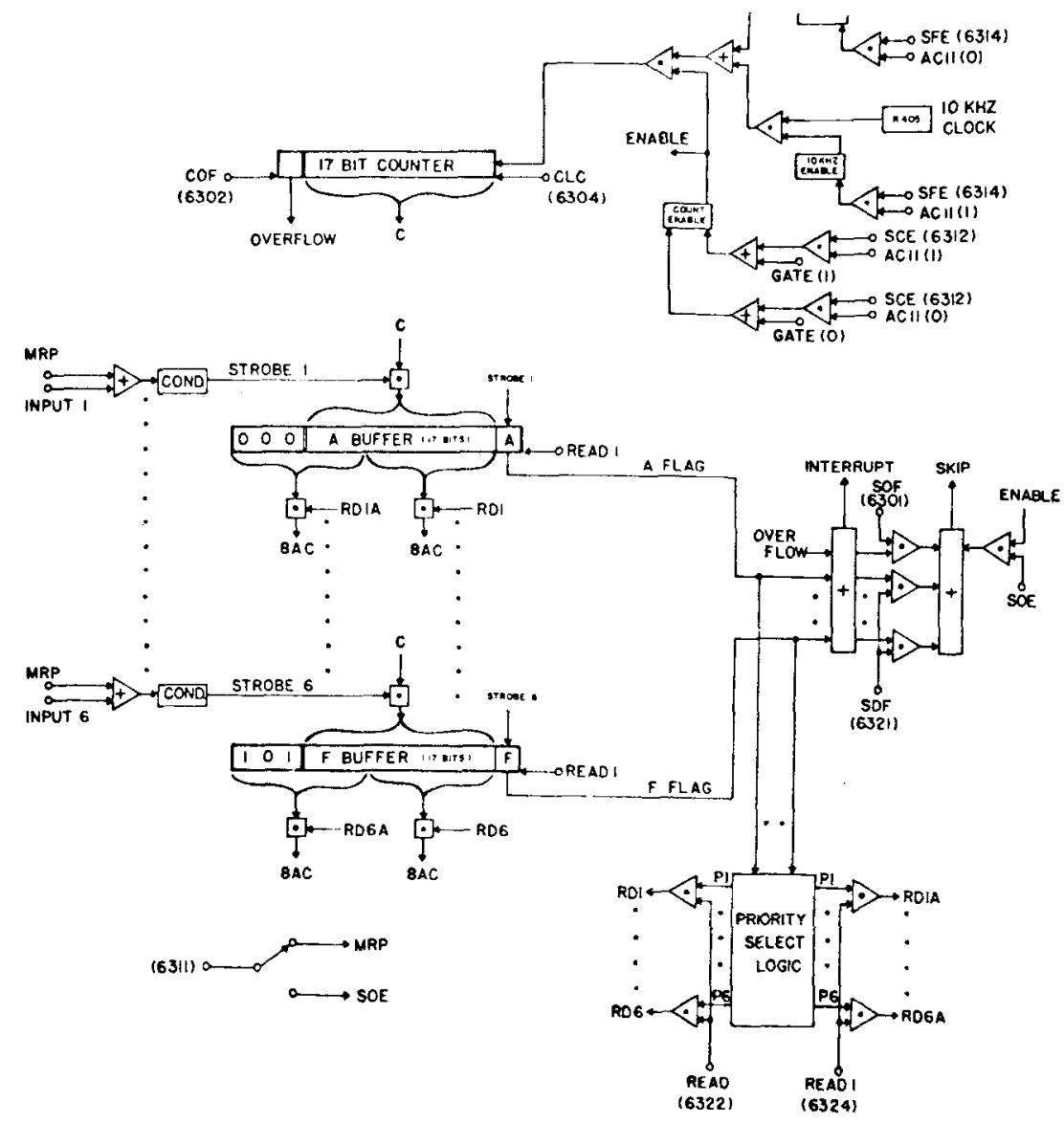

Fig. 1. Block diagram of interface. 
Table 1

Interface Instruction Set

\begin{tabular}{|c|c|c|}
\hline 6301 & SOF & $\begin{array}{l}\text { Skip on counter over- } \\
\text { flow flag. }\end{array}$ \\
\hline 6302 & COF & $\begin{array}{l}\text { Clear counter overflow } \\
\text { flag. }\end{array}$ \\
\hline 6304 & CLC & Clear counter. \\
\hline 6311 & $\begin{array}{l}\text { MRP* } \\
\text { SOE* }^{*}\end{array}$ & $\begin{array}{l}\text { Generate maintenance } \\
\text { pulse. } \\
\text { Skip if count enable } \\
\text { flip-flop is set. }\end{array}$ \\
\hline 6312 & SCE & $\begin{array}{l}\text { Set count enable flip- } \\
\text { flop if } A C 11=.1 \text {. } \\
\text { Clear count enable flip- } \\
\text { flop if } A C 11=0 \text {. }\end{array}$ \\
\hline 6314 & SFE & $\begin{array}{l}\text { Select } 10 \mathrm{KHz} \text { clock } \\
\text { if } \mathrm{AC11}=1 . \\
\text { Select alternate clock } \\
\text { if } \mathrm{AC11}=0 .\end{array}$ \\
\hline 6321 & SDF & Skip on data flag. \\
\hline 6322 & READ & $\begin{array}{l}\text { Read } 12 \text { least significant } \\
\text { bits into PDP- } 8 \text { accum- } \\
\text { ulator. }\end{array}$ \\
\hline 6324 & READ1 & $\begin{array}{l}\text { Read } 5 \text { most significant } \\
\text { bits and channel identi- } \\
\text { fication into PDP-8 } \\
\text { accumulator; clear the } \\
\text { data flag. }\end{array}$ \\
\hline
\end{tabular}

*Mechanically switched.

in software routines developed to diagnose malfunctions in the interface.

A recent modification allows the instruction which generates a maintenance pulse to be used in an alternate mode. In this mode the instruction acts as a skip instruction to test whether or not the interface is enabled. This test is particularly useful when an external enable voltage is used, since it allows the software to determine the state of the interface.

The interface consists of $128 \mathrm{R}$ - and $W$-series DEC modules contained in two Type 1943 mounting panels. This includes all of the logic, I/O cabling, and connectors to the experiment. Power for the logic is provided by a Type 728 power supply. The interface has been in operation for more than 18 months without a single malfunction.

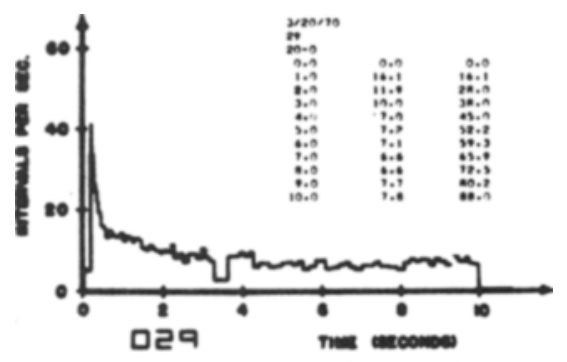

Pig. 2. Typical digital plotter output of instantaneous frequency vs time for 10-sec light flash. Inset: Summary data produced on Teletype.
The interface instruction set is shown in Table 1.

\section{APPLICATIONS}

The interface is currently being used in electrophysiological investigations of information processing in vision and audition, and in model studies of sensory systems. Some typical applications are briefly described below.

The functional organization of the visual system of the horseshoe crab (Limulus polyphemus) is being studied in terms of neural interactions between receptor units (Barlow, 1969). A fiber optics illuminator is used to stimulate a single receptor in the faceted eye of the animal. Impulses are being recorded from single nerve cells in the optic nerve in response to such stimulation. The firing rate of these cells is relatively low, usually less than $100 \mathrm{impulses} / \mathrm{sec}$. One or more channels of the interface are used, and the clock rate is $10 \mathrm{KHz}$. This allows measurement of times of occurrence with a resolution of $0.1 \mathrm{msec}$. The 17-bit counter overflows once each $13.1 \mathrm{sec}$, a fact which is taken account of in the software. The interface is controlled by an external gate signal derived from the stimulus timing source. Processed data is fed back to the $E$ following each stimulus presentation. A typical sample, shown in Fig. 2, includes a plot of instantaneous frequency vs time, the number of interimpulse intervals occurring during each 1 -sec period, and the accumulated number of intervals.

In a second application, the interface is being used off line to process neural impulses recorded from the auditory nerve cells in the ear of the Southern Army Worm Moth, Prodenia eridania, Cramer (Adams, 1969). One channel of the interface is used, but the clock rate is increased to $100 \mathrm{KHz}$. The interimpulse intervals are determined by resetting the $17-$ bit counter following arrival of each nerve impulse. The resulting interimpulse intervals are stored directly in core. As the data are being collected, an interval histogram is generated and continuously displayed. Such a display is shown in Fig. 3. Under console control it is possible to display the data as a joint interval histogram. The means of the joint interval can also be displayed (Rodieck, Kiang, \& Gerstein, 1962). Samples of these displays are shown in Figs. 4 and 5.

In still another application, the interface collects data from a number of analog models of auditory neurons (Kletsky \& Fraioli, 1967). The models are part of a real-time analog model of the peripheral auditory systems of

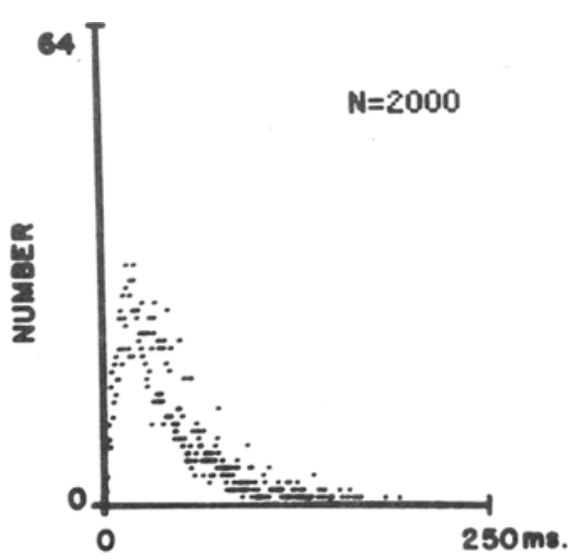

INTERVAL LENOTH

Fig. 3. Typical interval histogram of spontaneous neural activity.

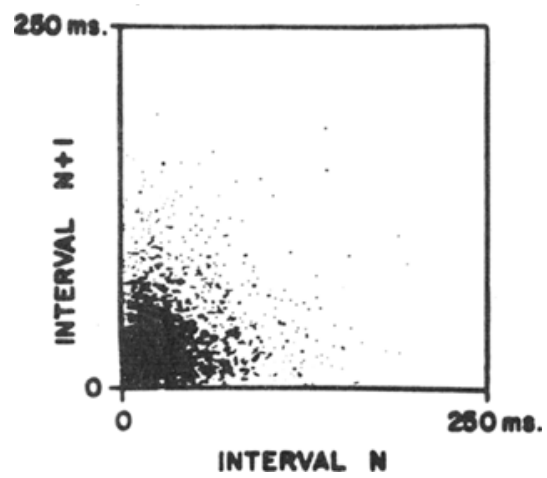

Fig. 4. Joint interval density histogram for data of Fig. 3.

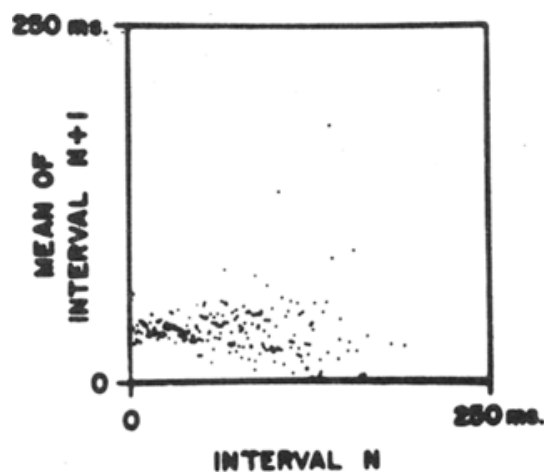

Fig. 5. Means of joint interval density histogram.

mammals. Data are collected as described for the moth experiments, and both interval and joint interval histograms can be generated. Since the firing rate of single neurons in the auditory system can exceed 500 impulses/sec, the $100-\mathrm{KHz}$ clock is used to provide adequate temporal resolution. In a portion of this work, 
GATE TIHE=IIUUి WII SEL:

CHAMHEL

\section{RATE}

$\begin{array}{ll}0 & 0065.35 \mathrm{FF} \\ 1 & 0064.45 \\ 2 & 0112.25 \mathrm{FF} \\ 3 & 0091.6 \mathrm{FF} \\ 4 & 0145.61 \mathrm{FF}\end{array}$

Fig. 6. Typical display of firing rates recorded simultaneously from five model neurons.

five channels of the interface are user to provide simultaneous numerical displays of firing rates determined from five individual neurons or neuron models. Both the time at which averaging begins and the time over which the averaging takes place can be controlled by an external gate signal. A typical display is shown in Fig. 6.

\section{SUMMARY}

$A$ versatile interface for the PDP- 8 has been described which allows the computer to accept times of occurrence of nerve impulses from physiological or real-time analog simulation experiments. The interface provides a means for efficiently handling the large amount of data produced in electrophysiological studies in vision and hearing.

\section{REFERENCES}

ADAMS, W. B. Intensity functions of auditory nerve fibers in the noctuid moth. Abstracts of the Third International Biophysics Conference, Cambridge, Mass., 1969

BARLOW, R, B., JR. Inhibitory fields in the Limulus lateral eye. Journal of General Physiology, 1969, 54, 38 3-396.

KLETSKY E. J., \& FRAIOLI, A. J. An electronic neuron model for sensory system studies. Proceedings of the National Electronics Canference. Toronto, Canada, 1967. Pp. 66-67.

RODIECK, R. W., KIANG, N. Y, S., \& GERSTEIN, G. L. Some quantitative methods for the study of spontaneous activity of single neurons. Biophysical Journal, 1962, 2, 351-368.

\title{
A PDP-12 system for on-line acquisition of heart rate data*
}

\author{
JOYCE KERR, MICHAEL TOBIN†, NORMAN MILKMAN†† \\ ZAVEN KHACHATURIAN, THOMAS WILLIAMS, and JOSEPH SCHACHTER \\ University of Pittsburgh School of Medicine, Pittsburgh, Pennsylvania 15213
}

A research program for investigating the responsiveness of infants at high risk for developing schizophrenia is described briefly. Two measures of reactivity to brief auditory stimuli, the evoked heart-rate response and the evoked potential of the brain, are recorded for analysis by the PDP-12 computer. An interface to the PDP-12, called the $R$ peak detector and $R-R$ interval counter, is used for reducing the EKG to $R-R$ intervals, with an accuracy of $\pm 1 \mathrm{msec}$. The current usage of the PDP-12 and its IBM-compatible tape transports is discussed, and proposed developments for on-line statistical analyses of the physiological data and real-time control of the experiment are presented.

\section{RESEARCH PROGRAM}

The Research Center in Child Psychiatry of the Pittsburgh Child Guidance Center and the University of Pittsburgh is undertaking a program to investigate the psychophysiology of infants who may be predisposed to schizophrenia. It is known that genetic factors play a role in the etiology of schizophrenia, and it is possible that those genetic factors may be influencing the functioning of the central nervous system at birth. By comparing the responsiveness of neonates at high risk for schizophrenia to that of neonates at low risk for the mental disorder, we hope to show the presence of differences in nervous system functioning before extrauterine environmental influences become important factors in the development of the illness.

*This work has been supported by grants from the $R$. $K$. Mellon Foundation S. Mellon Scaife Foundation, M. Falk Medical Fund, Buhl Foundation, and the Grant Foundation.

tNow at the New York State Psychiatric Institute, New York, New York.

H+Now at Rockefeller University, New York, New York.
In the Laboratory of Human Developmental Psychobiology, neonates are tested on the second and third days of life for a 4-h session each day. A brief auditory stimulus, consisting of a single cycle of a $1,200-\mathrm{Hz}$ tone, is delivered to the infant through loudspeakers at intervals of 30 to 45 sec. Fifty trials at each of three intensities and 50 control, or no-stimulus, trials are presented over a background of random noise.

Two measures of reactivity to the auditory stimuli, the evoked heart-rate response and the evoked potential of the brain, are recorded by the PDP-12 for statistical analysis. The EKG, EEG, and timing signals are also recorded on analog tape, permitting subsequent computer analysis. In addition, the EKG, EEG, EMG, EOG, respiration, and cardiotachometer output are monitored on a polygraph throughout the test session. These polygraph recordings can be used for relating the evoked potential and heart-rate responses to the overall state of the infant, or to sleep stage.

\section{HEART-RATE DATA}

Earlier work on the evoked heart-rate response in infants (Schachter et al, in press), which used computer conversion of EKG records, showed that a more efficient method for reducing the EKG to R-R intervals was necessary. Standard cardiotachometers can be used to provide the interval between $R$ peaks, but the accuracy of these instruments is $\pm 5 \%$ in terms of beats/minute. At a neonatal heart rate of $120 \mathrm{bpm}$, this corresponds to $a \pm 25$-msec variation in the R-R interval, a level of accuracy which is inadequate for measuring the small but statistically significant neonatal evoked heart-rate response. An alternative method of obtaining $R-R$ intervals would be to sample the EKG continuously with an A/D converter and to identify the $R$ peaks by computer programming, but we wished to save the power of our small laboratory computer for recording and analyzing other physiological responses simultaneously.

Therefore, an interface to the PDP-12, called the $R$ peak detector and $R-R$ interval counter, was designed to reduce EKG data to $R \cdot R$ intervals with an accuracy of $\pm 1 \mathrm{msec}$ (Tobin et al, 1970). Unlike standard cardiotachometers, the accuracy of this instrument is relatively independent of the frequency and amplitude of the incoming data. The reliability of the $R$ peak detector has been checked by comparing repeated readings of EKG signals recorded on analog magnetic tape. In addition, pulses from a function generator were fed into the instrument at known time intervals, and the readings of the $R$ peak detector were printed out on the PDP-12 Teletype. In both cases the measurement error did not exceed $\pm 1 \mathrm{msec}$. The validity of the instrument was evaluated by 